\title{
A propos des relations entre nature et ville \\ Bilan des dialogues \\ franco-lusophones de Tours: es questionnements pour des recherches futures!
}

\author{
Jean-Paul Carrière*
}

\begin{abstract}
Resumo Les journées franco-lusophones organisées à Tours du 13 au 15 juin 2018, et dédiées à la question des rapports entre nature et ville, s'inscrivent dans le cadre du programme "Attilio" et de la cinquième édition des dialogues en aménagement et urbanisme. Dans la première partie de ce texte, on rappelle la genèse de ces rencontres, pour ensuite présenter les axes thématiques abordés lors de ces journées. Cette présentation peut être lue comme une invitation adressée aux chercheurs en urbanisme des deux aires culturelles et linguistiques de la francophonie et de la lusophonie à continuer à explorer ces axes de réflexion.
\end{abstract}

Mots-clefs: dialogues franco-lusophones en urbanisme, relations ville - nature, axes de recherche.

\section{A propósito das relações entre natureza e cidade. Balanço dos dialogos franco-lusofonos de Tours: questões para pesquisas futuras!}

Resumo As jornadas franco-lusófonas dedicadas ao assunto das relações entre natureza e cidade, foram organizadas em Tours nos dias 13 a 15 de junho de 2018, no âmbito do programa "Attilio" e da quinta edição dos diálogos em planejamento e urbanismo. Na primeira parte desse texto recordamos a gênese desses encontros, antes de, na segunda parte, apresentar os eixos temáticos tratados dentro dessas jornadas. Este artigo pode ser encarado como um convite para os pesquisadores em urbanismo das duas áreas culturais e linguísticas da francofonia e da lusofonia seguirem explorando esses eixos de reflexão.

Palavras-chave: diálogos franco-lusófonos em urbanismo, relações de pesquisa cidade-natureza, questões de pesquisa.

\begin{abstract}
About the relationship between nature and city. Review of the franco-lusophone dialogues in Tours: questions for future research!
\end{abstract}

\begin{abstract}
The Franco-Lusophone meetings organized in Tours from June 13 to 15,2018 , and dedicated to the issue of relationships between nature and city, are part of the "Attilio" programme and the fifth edition of the dialogues on urban planning. In the first part of this text, we recall the genesis of these meetings, and then present the thematic axes addressed during these days. This presentation can be read as an invitation to urban researchers from the two cultural and linguistic areas of the Francophonie and Lusophony to continue exploring these topics.
\end{abstract}

Keywords: franco-lusophone dialogues in urban planning, relationships city - nature, lines of research. 
Du 13 au 15 juin 2018, s'est déroulé à Tours, en France, un colloque franco-lusophone organisé par l'Unité Mixte de Recherche du CNRS CITERES (Cités, Territoires, Société) de I'Université de Tours, en partenariat avec le Département Aménagement et Environnement de Polytech'Tours, sur le thème "Nature et Ville. Regards croisés franco-lusophones". Ce numéro spécial de Risco revient sur ce thème en rassemblant une sélection partielle des communications présentées en cette occasion, revues et actualisées, et complète une autre publication en cours de parution, qui prendra la forme d'un ouvrage en langue française publié par les Presses Universitaires François Rabelais, sous le titre: "Faire nature en ville".

Afin de mieux apprécier les apports de ce numéro spécial de Risco, il convient de rappeler ici la genèse des échanges franco-lusophones en urbanisme, développés dans le cadre du projet "Attilio", du nom du premier docteur en urbanisme brésilien à avoir soutenu sa thèse de doctorat en France, avant de pouvoir présenter les axes de recherche liés au thème central du colloque et de ce numéro de revue, qui en est I'un des prolongements.

\section{La genèse des "diálogos"}

Depuis onze ans, des chercheurs brésiliens et français en urbanisme et aménagement du territoire ont établi des liens de coopération et d'échange afin de réfléchir ensemble aux enjeux contemporains de la ville et à ses défis futurs. Des Dialogues franco-brésiliens en aménagement et urbanisme, communément désignés par le terme portugais de Diálogos se sont tenus alternativement dans les deux pays : Salvador/Bahia (2009, 2016), Paris (2011), São Paulo (2012), Lille (2014), Tours (2018).

Ces Diálogos sont animés par les deux associations qui rassemblent chacune les instituts d'urbanisme et aménagement francophones et brésiliens, I'APERAU Internationale, Association pour la Promotion de l'Enseignement et de la Recherche en Aménagement et Urbanisme, et I'ANPUR, Associação Nacional de Pósgraduação e Pesquisa em Planejamento Urbano e Regional. Toutefois, le principe de l'élargissement des Diálogos aux deux aires culturelles de la francophonie et la lusophonie a été arrêté lors de la rencontre de Lille en 2014 et réaffirmé en juin-juillet 2016, à l'occasion de la quatrième édition de Salvador de Bahia et lors du World Planning Schools Congress de Rio de Janeiro, également en 2016.

* Jean-Paul Carriére est Économiste, Professeur émérite en Aménagement de l'espace et urbanisme, Unité Mixte de Recherche CNRS 7324 CITERES, Université de Tours, France, ORCID <https://orcid.org/00000002-5781-4369>.
L'objectif de cet élargissement, dont le colloque de Tours a été la première manifestation, était de promouvoir, à l'échelle des deux aires culturelles et linguistiques, les échanges scientifiques relatifs aux enjeux contemporains posés, au nord comme au sud, par la ville, son organisation socio-spatiale et sa dynamique de développement. II s'agit pour les promoteurs de ces échanges de nourrir le débat scientifique sur le devenir des villes, confrontées aux défis globaux et locaux, que ceux-ci soient climatiques, sociaux, économiques, culturels, et de partager des résultats de recherches concernant 
aussi bien les pays africains que européens ou américains du nord comme du sud, appartenant aux deux aires en question.

Les pays et territoires qui s'y trouvent représentent à coup sûr un éventail très large de situations urbaines, propice aux comparaisons et à l'échange d'expériences socialement significatives, et c'est, forts de cette conviction, que les organisateurs de la dernière édition des Diálogos ont retenu, dans une perspective interdisciplinaire large allant des sciences de la nature aux sciences humaines et sociales, le thème des rapports entre nature et ville, thème devenu particulièrement prégnant en urbanisme, à travers le monde quelles que soient les spécificités des contextes nationaux et locaux.

\section{Les rapports nature-ville : des questionnements multiples au coeur des Diálogos 5}

Le thème du colloque de Tours renvoie à une multiplicité de questionnements multidimensionnels, pour certains fort anciens et pour d'autres émergents.

Dans un contexte fortement marqué par des perturbations globales allant du changement climatique aux mutations urbaines profondes, liées notamment au phénomène de la métropolisation induit par la globalisation, de nombreuses questions ont surgi ces dernières années qui procèdent d'une interrogation plus générale sur le contenu et les évolutions des rapports entre ville et nature. Au demeurant, les impacts de la globalisation et les perturbations globales (modification du climat, raréfaction des ressources, diminution de la biodiversité, standardisation des paysages ...) s'expriment également à travers des impacts directs et indirects sur les villes intermédiaires, moyennes et petites.

C'est ce qui explique l'importance prise ces dernières années par un questionnement général sur la réalité des rapports de complémentarité et/ou d'opposition entre ville et nature. Ceci se vérifie dans les pays appartenant aux aires culturelles de la francophonie et de la lusophonie, mais avec des approches souvent différentes, les différences tenant à la fois au contexte sociétal, aux trajectoires du développement urbain et à la diffusion de modèles de "nature urbaine" spécifiques (on peut penser ici, par exemple, à l'influence considérable au Brésil d'un Burle Marx sur la façon d'intégrer la nature dans l'espace public des villes).

Aborder la question des rapports ville-nature, telle qu'elle se pose dans les deux aires culturelles et dans les pays qui s'y rattachent, ne doit pas conduire à faire abstraction des spécificités internes à ces deux aires et à ignorer la diversité des approches que l'on peut rencontrer de part et d'autre, que I'on soit au Maroc, au Brésil, au Québec, en Belgique ou au Mozambique, en Suisse ou dans tout autre pays luso- ou francophone, comme on peut le constater au fil des articles rassemblés dans ce numéro. En outre, par son ampleur, cette question posée en priorité aux aménageurs-urbanistes, fait appel aux apports de nombreuses disciplines voisines, comme l'Écologie urbaine, l'Écologie du paysage, la Géographie, la Sociologie, l'Économie, la Science politique ou encore l'Architecture, dans une perspective d'interdisciplinarité.

La question centrale des rapports entre nature et ville peut se décliner selon trois axes thématiques de recherche principaux retenus par l'appel à communication du 
colloque de Tours. Nous les rappelons ici, en reprenant en partie le texte de l'appel à communications, et en invitant les chercheurs en urbanisme à continuer à les explorer, car de toute évidence, ce n'est pas en un seul colloque que l'on pourra épuiser le thème général de la relation ville-nature.

\section{- 1er axe : Les dynamiques des rapports ville-nature}

Cet axe intègre une liste de questions qui concernent à la fois les incidences de I'urbanisation, des activités urbaines, et de l'aménagement, sur les écosytèmes. De quelle façon ceci amène-t-il à imaginer la ville autrement ? Participent de cet axe des réflexions et analyses sur l'étalement urbain, ses modalités et ses impacts sur les espaces naturels et agricoles et la dynamique des milieux (terrestres ou aquatiques), sur la place de la nature dans le design urbain et plus généralement le paysage urbain et périurbain, sur la création des corridors écologiques (tels qu'en France les Trames Vertes et Bleues) et des actions visant le maintien d'espaces naturels en ville.

Par leurs dynamiques propres, les espaces agricoles ou les réseaux écologiques imposent des contraintes aux espaces urbanisés, ce qui remet en cause la conception et la forme de ceux-ci. En outre, l'espace urbain ne peut être considéré comme un "désert écologique" : les fronts d'eau, les bords de fleuve, les délaissés, les friches, les espaces forestiers ou agricoles sont autant d'écosystèmes intra-urbains, certes soumis à une forte incidence anthropique, mais abritant néanmoins une biodiversité importante. La compréhension de ces espaces à l'échelle locale ou plus globale ouvre des perspectives pour de nouvelles méthodes de préservation des espaces naturels mais aussi de valorisation touristique.

\section{- 2ème axe : La nature, atout ou menace pour la ville ?}

La nature est souvent perçue comme ressource à mobiliser pour un mieux vivre ensemble en ville. La notion de service écosystémique peut être ici convoquée pour analyser comment les espaces non bâtis et végétalisés, les espaces agricoles résiduels ou encore les milieux aquatiques, sont pris en compte dans les plans et projets d'aménagement. Il s'agit de dépasser une approche strictement écologique de la nature pour s'interroger sur les fonctions multiples qu'elle peut remplir dans un contexte urbain (amélioration du bien-être et de la santé, esthétique, espace nourricier, espace de rencontre et de fabrication de lien social, protection face au risque ou à la formation d'ilôts de chaleur...). Par ailleurs, la vision d'une nature-ressource peut aussi être posée dans une approche patrimoniale, comme bien commun à préserver ou à restaurer.

Toutefois, la nature peut également être associée aux menaces qu'elle fait peser sur la ville, inhérentes à l'intensité plus ou moins forte de risques divers, tels que les inondations, le changement climatique, les séismes... Ces menaces, d'autant plus fortes qu'elles peuvent parfois se conjuguer avec de forts risques industriels, appellent en retour une réflexion sur l'adaptation de la ville aux risques "naturels" à travers des actions d'aménagement, que ce soit dans une perspective d'atténuation du risque ou de réduction de la vulnérabilité de la ville, qui peut aller jusqu'à repenser le modèle de développement urbain.

"l y a donc là un axe de réflexion à part entière qui concerne une nature urbaine "Janus", aux deux visages. 


\section{- 3ème axe : Nature vécue et nature agie : représentations vs action publique}

Peut-on parler d'une congruence ou au contraire d'un hiatus entre les perceptions et représentations des rapports ville - nature d'une part, et les actions et stratégies des acteurs, publics et privés, de la "fabrique de la ville", d'autre part ?

En premier lieu se pose la question de l'émergence d'un "désir de nature" et de la formation d'une demande sociale de nature chez les habitants ou les usagers au sein des deux aires culturelles; ce qui conduit à s'interroger sur les perceptions et les représentations de la nature, chez les habitants comme chez les producteurs du cadre bâti. Peut-on à cet égard discerner des différences de perspective ou au contraire des communautés de vision, qui induiraient différentes façons "d'instrumentaliser" la nature dans les opérations et projets d'aménagement urbain ?

Mais, en second lieu, se pose également la question des impacts de la "mise en nature" de la ville sur son organisation socio-spatiale. Quels en sont les effets en termes de fragmentation urbaine, de gentrification, et d'inégalités d'accès à la nature et ses services ? Quelles sont les incidences des projets d'aménagement destinés à valoriser la nature en ville (aménagement de parcs, de berges de rivière et de fronts d'eau, de trames vertes et bleues, etc.) sur l'appropriation de l'espace urbain par les catégories sociales ? sont-elles inclusives, ou favorisent-elles de nouvelles discriminations sociospatiales ? Quels rôles jouent les acteurs de la production urbaine, en particulier le capital immobilier, dans la conception et la mise en œuvre de ces projets. Ce type de question nous renvoie plus globalement à des interrogations sur ce que peut recouvrer la notion de "justice environnementale urbaine" et la façon dont elle peut être prise en compte dans la conception et la mise en œuvre de l'action urbanistique.

Un autre volet de cet axe concerne enfin les modalités-mêmes de l'action sur la nature urbaine : sur les plans institutionnels et politiques, quels sont les facteurs qui président à l'élaboration d'une politique ou d'un projet de préservation de la nature ou de renaturation en ville ? Quels sont les obstacles rencontrés ? Voit-on émerger à travers politiques et projets de nouveaux modèles de développement urbain circulant à l'échelle internationale, pouvant aller jusqu'à de véritables ruptures dans les pratiques passées ?

S'interroger sur les rapports ville - nature, dans une perspective praxéologique, ne peut faire non plus l'économie d'une réflexion sur la place de la nature dans les utopies urbaines, tout comme sur le rôle qu'elle peut jouer dans la construction, planifiée ou non, d'une identité urbaine. II est d'ailleurs aisément vérifiable que la nature et le paysage sont de plus en plus souvent présents au cœur du projet urbain, les paysagistes étant directement sollicités pour son élaboration et consultés pour l'élaboration des documents d'urbanisme de l'échelle régionale à l'échelle locale. Que peut-on en déduire quant aux mutations de l'urbanisme contemporain ?

Dès lors que l'on évoque les stratégies urbaines et l'action publique, la question de la gouvernance occupe une place essentielle : Quelles sont les nouvelles formes de gouvernance induites par la prise en compte de la nature urbaine dans l'action publique locale ? En quoi les projets de nature en ville sollicitent-ils ou entraînent-ils plus de participation citoyenne, sous des formes nouvelles ? Y-a-t-il de nouveaux acteurs à prendre place sur la scène de l'action publique? 
Enfin, dès lors que la nature en ville incorpore le champ d'action des urbanistes, on ne peut manquer de s'interroger sur son statut de "bien commun", alors que les espaces dits "naturels" sont de plus en plus soumis à des processus de privatisation, via l'action des groupes immobiliers. On peut à cet égard se demander jusqu'à quel point il peut y avoir "instrumentalisation" de la "nature" à des fins d'attractivité et de compétitivité urbaine à travers différentes pratiques telles que, les éco-quartiers, les opérations de verdissement, la valorisation et l'aménagement d'espaces naturels, la recherche "d'aménités vertes", etc.

Au total, cet axe renvoie à une multiplicité d'interrogations qui concernent aussi bien la dimension substantielle de l'action, celle qui touche au contenu de l'intersection des trois piliers du développement durable, que sa dimension procédurale.

L'ensemble des questions évoquées à travers ces trois axes peuvent être abordées à différentes échelles, que ce soit celle de l'ilôt et du quartier, du cadre de vie de I'habitant, à celles de la ville, de l'agglomération, et plus largement à celle de l'aire fonctionnelle urbaine ou encore de la région métropolitaine, en tant que système urbain polycentrique. Quant à la nature, elle peut également être appréhendée sous différentes dimensions qui vont de la biodiversité au paysage, en passant par les espaces verts, les écoystèmes et les services qu'ils rendent.

Si ces questions avaient été explicitement énoncées dans l'appel à communication, il était cependant évident que le colloque ne pouvait les aborder toutes de façon exhaustive, et c'est pourquoi nous considérons qu'elles peuvent encore être au cœur de recherches futures, en particulier dans le cadre de partenariats franco-lusophones ; ne serait-ce que pour mettre à jour à la fois les invariants et les différences dans la façon d'appréhender - et d'agir sur - les rapports ville - nature dans les pays qui composent les aires culturelles francophones et lusophones, et au-delà de penser comment peuvent s'articuler les actions sur les espaces urbains et "naturels". 